\title{
PRECODING OF ORTHOGONAL STBC WITH CHANNEL COVARIANCE FEEDBACK FOR MINIMUM ERROR PROBABILITY
}

\author{
Yi Zhao, Raviraj Adve and Teng Joon Lim \\ Dept. of Electrical and Computer Engineering, University of Toronto, \\ 10 King's College Road, Toronto, ON, M5S 3G4, Canada. \\ E-mail: zhaoyi, rsadve, limtj@comm.utoronto.ca
}

\begin{abstract}
This paper develops the linear transformation (or precoding) of orthogonal space-time block codes (STBC) for minimizing probability of decoding error, when the channel covariance matrix is available at the transmitter. We build on recent work that stated the optimization problem without solving for the transformation. Specifically, we provide a closed-form solution for the multi-input single-output (MISO) systems, and a numerical solution for the multiinput multi-output (MIMO) systems. Our results confirm that eigen-beamforming is optimal at low SNR or highly correlated channels, and full diversity is optimal at high SNR or weakly correlated channels.
\end{abstract}

Keywords - MIMO systems, STBC.

\section{INTRODUCTION}

In mobile communications, the adverse effects of channel fading can be mitigated by transmission over a diversity of independent channels. A large and growing body of results have firmly established the potential of orthogonal spacetime block codes (O-STBC) in multi-input multi-output (MIMO) systems that use antenna arrays at the transmitter and the receiver [1] to provide spatial diversity at both ends of a communications link.

Without channel state information (CSI) at the transmitter, space-time codes should be designed using the rank and determinant criteria, even when the spatial channels are correlated. It is known that spatial correlation results in a smaller coding advantage [1]. But given that nothing can improve the performance of current state-of-the-art fulldiversity space-time codes without CSI at the transmitter, it is natural to consider performance improvements when this assumption is relaxed.

In this paper, we study the design of a linear precoder for O-STBC in spatially correlated, quasti-static, flat fading channels with channel covariance knowledge available at the transmitter. The objective is to minimize the average probability of decoding error. Prior work on this topic developed the optimality criterion [2] to be satisfied by the precoding matrix, but no closed-form or numerical solution was provided. Recent study in [3], [4] solved the optimal precoding matrices for MISO systems and particular MIMO systems with uncorrelated receive antennas. In this paper, we present a numerical solution for arbitrary MIMO channels with both transmit and receive correlations. We can see our solution contains the ones in [3], [4] as special cases.

Furthermore, this problem setting ties in with recent work on determining the capacity-achieving signal correlation matrix when the channel covariance is available at the transmitter [5], [6]. In contrast, our research is focused on minimizing the error probability, given a linear precoding structure based on O-STBC. This transmitter structure is chosen mainly to simplify the transmitter and the receiver complexity. Due to the orthogonal code structure, the complexity is only linear in the number of antennas despite employing a maximum likelihood receiver.

The rest of the paper is organized as follows: Section II presents the background material needed in the rest of the paper, Section III discusses the optimal precoding under various scenarios, Section IV introduces two simplified strategies that are shown to result in minimal performance loss. Simulation examples are presented in Section V. Finally, conclusions are given in Section VI.

\section{BACKGROUND}

Consider a MIMO system with $M$ transmit and $N$ receive antennas. O-STBC is used, and a linear transformation $\mathbf{W} \in \mathbb{C}^{M \times M}$ is applied prior to transmission to improve performance in correlated fading. The transmitter for such a system is shown in Fig. 1. A maximum-likelihood (ML) receiver is employed. Our goal is to find the optimal W to minimize the maximum pairwise error probability (PEP) between codewords.

The MIMO channel is described by the $N \times M$ matrix $\mathbf{H}$ where the element $[\mathbf{H}]_{n m}$ is the fading coefficient between the $m$ th transmit antenna and the $n$th receive antenna. The channel correlation matrix is

$$
\mathbf{R}=E\left[\mathbf{h h}^{\dagger}\right]
$$

where $(\cdot)^{\dagger}$ denotes Hermitian transpose, $\mathbf{h}=\operatorname{vec}(\mathbf{H})$, and $\operatorname{vec}(\cdot)$ denotes the vectorization operator which stacks the columns of $\mathbf{H}$.

The STBC encoder organizes data into an $M \times L$ matrix $\mathbf{C}$ and successive columns of this matrix are transmitted over $L$ time indices. The corresponding $N \times L$ received signal 


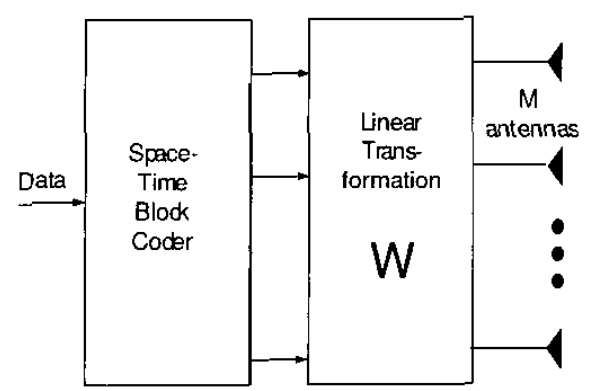

Fig. 1. Precoded STBC transmitter block diagram.

matrix $\mathrm{X}$ can be written as

$$
\mathbf{X}=\mathbf{H W C}+\mathbf{E} \text {, }
$$

where $\mathbf{E}$ is an $N \times L$ matrix with i.i.d. complex Gaussian elements representing additive thermal noise. The receiver employs an ML decoder, thus the decoded codeword $\widehat{\mathbf{C}}$ can be expressed as

$$
\widehat{\mathbf{C}}=\arg \min _{\mathbf{C}}\|\mathbf{X}-\mathbf{H W C}\|_{F}^{2},
$$

where $\|\cdot\|_{F}$ is the Frobenius norm [7]. Note that, because HW is equivalent to a modified channel matrix $\tilde{\mathbf{H}}$, maximum likelihood decoding of $\mathbf{C}$ requires only the simple linear operation described in [1].

In [2], it was found that the $\mathbf{W}_{\text {opt }}$ which minimizes the maximum pairwise error probability, i.e.

$$
\mathbf{W}_{\text {opt }}=\arg \min _{\mathbf{W}} \max _{\mathbf{C}_{k}, \mathbf{C}_{l}} P\left(\mathbf{C}_{k} \rightarrow \mathbf{C}_{l}\right)
$$

satisfies

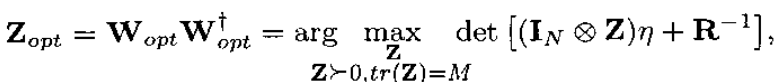

where $\mathbf{Z}$ has to be positive semi-definite because $\mathbf{Z}=$ $\mathbf{W} \mathbf{W}^{\dagger}$, and the trace constraint is necessary to avoid power amplification. $\otimes$ denotes the Kronecker product, and $\eta=$ $\mu_{\min } / 4 \sigma^{2}$ with

$$
\mu_{\min }=\arg \min _{\mu_{k l}}\left\{\mu_{k l} \mathbf{I}=\left(\mathbf{C}_{k}-\mathbf{C}_{l}\right)\left(\mathbf{C}_{k}-\mathbf{C}_{l}\right)^{\dagger}\right\}
$$

\section{OPTIMAL TRANSFORMATION}

\section{A. General Solution}

It is reasonable to assume that the correlation between two subchannels is equal to the product of the correlation at the transmitter and that at the receiver [8]. In matrix form, letting $\mathbf{R}_{T}$ denote the correlation between different transmit antennas, and $\mathbf{R}_{R}$ that between receive antennas, the channel correlation is

$$
\mathbf{R}=\mathbf{R}_{R} \otimes \mathbf{R}_{T}
$$

With this assumption, the optimal transformation matrix is the square root of

$$
\mathbf{Z}_{\text {opt }}=\underset{\mathbf{Z} \succeq 0, \operatorname{tr}(\mathbf{Z})=M}{\arg } \max _{\substack{\mathbf{Z}\\}} \operatorname{det}\left[\left(\mathbf{I}_{N} \otimes \mathbf{Z}\right) \eta+\mathbf{R}_{R}^{-1} \otimes \mathbf{R}_{T}^{-1}\right] .
$$

Theorem 1: Let $\mathbf{B}=\eta \mathbf{U}_{T}^{\dagger} \mathbf{Z} \mathbf{U}_{T}$, where $\mathbf{U}_{T}$ is the eigenmatrix of $\mathbf{R}_{T}$, i.e. $\mathbf{R}_{T}=\mathbf{U}_{T} \boldsymbol{\Lambda}_{T} \mathbf{U}_{T}^{\dagger}$. Solving (8) is equivalent to finding

$$
\mathbf{B}_{\text {opt }}=\arg \max _{\substack{\mathbf{B} \succeq 0 \\ \operatorname{tr}(\mathbf{B})=\eta M}} \operatorname{det}\left[\mathbf{I}_{N} \otimes \mathbf{B}+\boldsymbol{\Lambda}_{R}^{-1} \otimes \mathbf{\Lambda}_{T}^{-1}\right] .
$$

Proof: See Appendix.

Since $\Lambda_{T}$ and $\Lambda_{R}$ are diagonal, the solution to (9) must be diagonal as well. Let the $m$ th diagonal elements of $\Lambda_{T}$ and $\mathbf{B}$, and the $n$th diagonal element of $\Lambda_{R}$ be $\lambda_{t m}, b_{m}$ and $\lambda_{r n}$, respectively. The problem (9) is seen to be equivalent to finding a set of non-negative $b_{m}$ 's to maximize

$$
\prod_{m=1}^{M} \prod_{n=1}^{N}\left(b_{m}+\lambda_{r n}^{-1} \lambda_{t m}^{-1}\right)
$$

under the trace constraint $\operatorname{tr}(\mathbf{B})=\sum_{m} b_{m}=\eta M$. This problem is an extension of the waterfilling problem to two dimensions ( $m$ and $n$ ), so we can call it a secondorder waterfilling problem. The closed-form solution to this problem is too complicated to find. However, we can find the solution by numerical methods such as Sequential Quadratic Programming (SQP) [9]. Results of the numerical scheme are provided in Section $\mathrm{V}-\mathrm{A}$.

Since $\mathbf{Z} \eta=\mathbf{U}_{T} \mathbf{B} \mathbf{U}_{T}^{\dagger}$, the diagonal matrix $\mathbf{B}$ is actually the eigenvalue matrix of $\mathbf{Z} \eta$. Thus optimization results for $\mathbf{Z}$ and $\mathbf{W}$ are

$$
\begin{aligned}
\mathbf{Z} & =(1 / \eta) \mathbf{U}_{T} \mathbf{B} \mathbf{U}_{T}^{\dagger}, \\
\mathbf{W} & =(1 / \sqrt{\eta}) \mathbf{U}_{T} \sqrt{\mathbf{B} \boldsymbol{\Phi}} .
\end{aligned}
$$

where $\Phi$ can be any $M \times M$ unitary matrix, and the optimal $\mathbf{W}$ is not unique. For simplicity we can either choose $\mathbf{\Phi}=$ $\mathbf{I}_{M}$ or $\mathbf{\Phi}=\mathbf{U}_{T}^{\dagger}$. In this paper we choose the identity matrix.

\section{B. Closed-Form Solution for MISO systems}

We now consider the special case of MISO systems, i.e. systems with only a single receive antenna $(N=1)$. This is quite reasonable for the downlink of wireless communication systems since it may be too complicated and costly to employ more than one antenna at the mobile unit. Under this assumption the Kronecker product in (9) disappears and we end up solving

$$
\mathbf{B}_{\text {opt }}=\arg \max _{\substack{\mathbf{B} \succ 0 \\ \operatorname{tr}(\mathbf{B})=\eta}} \operatorname{det}\left[\mathbf{B}+\mathbf{\Lambda}_{T}^{-1}\right]
$$

where $\mathbf{B}$ is a non-negative diagonal matrix. This is identical to the water-filling problem in information theory [10], which has the solution

$$
b_{m}=\left(\nu-\lambda_{t m}^{-1}\right)^{+}, \quad \text { for } m=1, \ldots, M,
$$


where $\nu$ is a constant chosen to satisfy the trace constraint and $\mathbf{B}=\operatorname{diag}\left(b_{1}, \ldots, b_{M}\right)$. The notation $(x)^{+}$is used to denote the positive part of $x$, i.e. $(x)^{+}=x$, if $x>0$, and $(x)^{+}=0$, otherwise. The optimal $\mathbf{W}$ is

$$
\mathbf{W}_{\text {opt }}=\frac{1}{\sqrt{\eta}} \mathbf{U}_{T} \sqrt{\mathbf{B}_{\text {opt }}} .
$$

We can see that this solution agrees with those in [3], [4].

The transmitted signal $\mathbf{W}_{o p t} \mathbf{C}$ occupies the subspace spanned by the non-zero eigenvectors of $\mathbf{R}_{T}$. In the case of very high correlation, only one $b_{m}$ is non-zero, so we have eigen-beamforming. On the contrary, all the eigenvectors are used when the correlation is low, and full diversity is achieved.

\section{Relation to Capacity Analyses}

There has been much interest in the information theory community in MIMO channels with covariance feedback [5], [6]. In those works, the goal is to find the input covariance matrix $\mathbf{S}_{x, o p t}$ necessary to achieve ergodic channel capacity, while our goal is to find the optimal linear transformation to achieve minimum error probability. Interestingly, the conclusions reached are strikingly similar for both approaches, and warrants some comment.

1) Transmitting over the eigenvectors of the channel correlation matrix is optimal assuming only the channel correlation is available at the transmitter. However, the exact amount allocated to each eigen-channel may differ for the two schemes.

2) Beamforming is optimal at high correlation/low SNR. Diversity is not helpful in this case as it is noise and not fading that limits performance.

3) Optimal diversity order increases with SNR. At low SNR, only the strongest eigen-channel is used. As the SNR increases, more eigen-channels come into use, so the diversity order increases until full diversity is achieved.

4) Full diversity is optimal in uncorrelated channels. Similarly, in the high SNR region, the optimal scheme should use all the eigen-channels, because in this case diversity can be taken advantage of.

\section{SiMPLIFIED SCHEMES}

\section{A. Ignoring the Receive Correlation}

The extended "one ring" model introduced in [11] is a well known scattering model for channel correlation. From this model, for the downlink, the correlation of the fading coefficients between transmit antennas $p$ and $q$ and receive antenna $m$ is

$$
\mathbf{R}_{T}(p, q)=E\left[h_{m p} h_{m q}^{*}\right] \approx J_{0}\left(\Delta \frac{2 \pi}{\lambda} d^{T}(p, q)\right),
$$

where $\Delta$ is the angle spread, $\lambda$ is the wavelength, $d^{T}(p, q)$ is the distance between the two transmit antennas, and $J_{0}(\cdot)$ is the first kind Bessel function of the zeroth order. The correlation between two receive antennas $l$ and $m$ is

$$
\mathbf{R}_{R}(l, m)=E\left[h_{l p} h_{m p}^{*}\right]=J_{0}\left(\frac{2 \pi}{\lambda} d^{R}(l, m)\right),
$$

where $d^{R}(l, m)$ is the distance between the two receive antennas.

In practice, the angle spread $\Delta$ is usually very small. As a result, the receive correlation is small compared to the transmit correlation. For instance, if the distance between two transmit antennas equals $\lambda / 2$ and the angle spread is $\Delta=0.1$, the correlation between these two transmit antennas is $J_{0}(0.1 \pi)=0.97$. But the correlation between two receive antennas with the same separation is just $J_{0}(\pi)=$ -0.30 .

In dealing with receive diversity, a correlation below 0.5 is considered negligible [12]. Therefore we can simplify our algorithm for the downlink transmission by ignoring the receive correlation. Under this approximation, the rows of $\mathbf{H}$ are assumed independent and the channel correlation matrix can be written as $\mathbf{R}=\mathbf{I}_{N} \otimes \mathbf{R}_{T}$. In this case, (9) becomes

$$
\begin{aligned}
\mathbf{B}_{\text {opt }} & =\arg \max _{\substack{\mathbf{B} \succ 0 \\
\operatorname{tr}(\mathbf{B})=\eta M}} \operatorname{det}\left[\mathbf{I}_{N} \otimes \mathbf{B}+\mathbf{I}_{N} \otimes \mathbf{\Lambda}_{T}^{-1}\right] \\
& =\arg \max _{\substack{\mathbf{B} \succ 0 \\
\operatorname{tr}(\mathbf{B})=\eta M}} \operatorname{det}\left[\mathbf{B}+\mathbf{\Lambda}_{T}^{-1}\right]^{N}
\end{aligned}
$$

Therefore, the solution is exactly the same as in (14), and the complicated second-order water-filling is avoided.

\section{B. Switching Between Beamforming and STBC}

We can avoid waterfilling altogether by switching between beamforming and O-STBC at a pre-computed threshold SNR level. This threshold is found by equating the error probability performance criteria of beamforming and O-STBC. Specifically, we want to find $\eta$ that solves the equation

$$
\operatorname{det}\left[\mathbf{Z}_{\text {beam }} \eta+\mathbf{R}_{T}^{-1}\right]=\operatorname{det}\left[\eta \mathbf{I}_{M}+\mathbf{R}_{T}^{-1}\right] .
$$

where $\mathbf{Z}_{\text {beam }}$ is the $\mathbf{Z}$ matrix for beamforming, i.e. $\mathbf{Z}_{\text {beam }}=$ $\alpha \mathbf{u}_{t 1} \mathbf{u}_{t 1}^{\dagger}$ where $\alpha$ is a constant determined from (14) and $\mathbf{u}_{t 1}$ is the eigenvector corresponding to the largest eigenvalue of $\mathbf{U}_{T}$.

It is self-evident that the simplified strategy incurs a greater loss in performance relative to the full-complexity scheme when the transition region between beamforming and O-STBC grows. There are however cases when the transition region is so small that no difference in performance is discernible.

One example is when the correlation between antennas is low. In this case all the eigenvalues are close to 1 , so the transition region is small. Another example is when all the channel correlations are equal, in which case the eigenvalues 


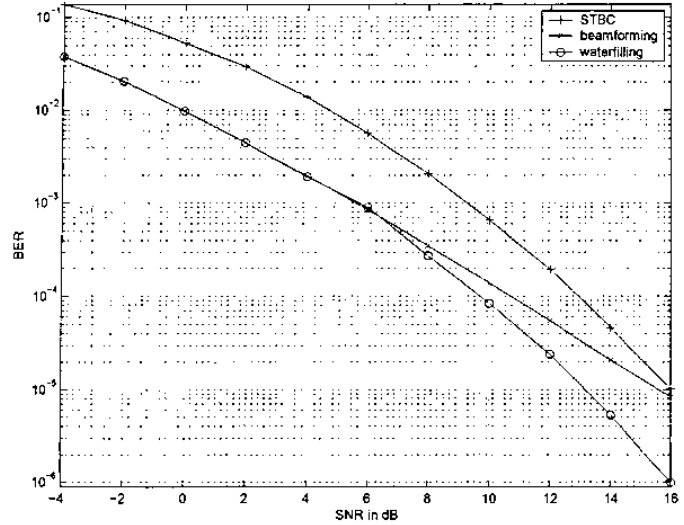

Fig. 2. Optimal scheme for MIMO system. $M=4, N=2$.

of $\mathbf{R}_{T}$ take on only two values so that the transition region has zero width. To show this, consider

$$
\mathbf{R}_{T}=\left(\begin{array}{cccc}
1 & \rho & \ldots & \rho \\
\rho & 1 & \ldots & \rho \\
\vdots & \vdots & \ddots & \vdots \\
\rho & \rho & \ldots & 1
\end{array}\right)
$$

This matrix has only two eigenvalues: $(1+\rho)$ and $(1-\rho)$ (repeated $(N-1)$ times). Therefore, the waterfilling scheme has no transition region. In the low SNR region, only the eigen-channel corresponding to $(1+\rho)$ is used, so we have beamforming. All the other $N-1$ channels will come into use simultaneously when the SNR exceeds the threshold level, so the performance is quite close to STBC. Therefore, the switching scheme can achieve very good performance under this correlation model.

\section{Simulation Results}

\section{A. Numerical Solutions for MIMO Systems}

As discussed in Section III-A, the optimal transformation for MIMO system is found through a second-order waterfilling problem. No closed form solution has been found, but numerical methods, such as SQP can be used to solve (10) with a trace constraint. Here we use the MATLAB function fmincon to solve the problem.

Figure 2 shows the performance curve obtained with the optimal transformation. The correlation matrix is

$$
\mathbf{R}_{T 41}=\left(\begin{array}{cccc}
1 & 0.9755 & 0.9037 & 0.79 \\
0.9755 & 1 & 0.9755 & 0.9037 \\
0.9037 & 0.9755 & 1 & 0.9755 \\
0.79 & 0.9037 & 0.9755 & 1
\end{array}\right)
$$

It is obtained by using (16) from the extended "one ring" model. The distance between two adjacent antennas is $\lambda / 2$, and the angel spread is $\Delta=0.1$ radians. Similarly, the correlation between the two receive antennas is set to be

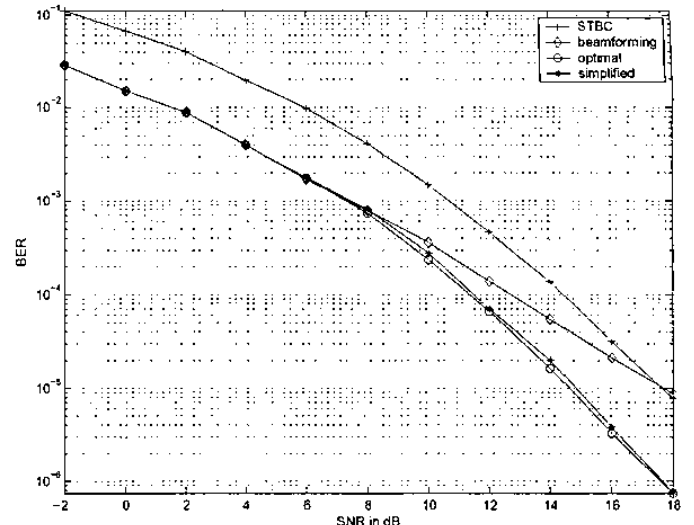

Fig. 3. BER curves when receive correlations are ignored.

$$
M=4, N=2 \text {. }
$$

-0.3042 based on (17). From the plots we can see that for very low SNR, the optimal transformation is equivalent to beamforming, as expected. For the other SNR regions, the performance of the optimal scheme is better than both beamforming and STBC. Furthermore, the optimal scheme approaches STBC as SNR increases, again as expected.

\section{B. Simplified Schemes}

Figure 3 shows the performance when we ignore receiver correlation. A system with four transmit and two receive antennas is considered. The transmit correlation is given in Eqn. (20). At the receiver side, the correlation between the two receive antennas is set to a very high value of 0.7 . From the figure we can find that there is nearly no performance loss for ignoring the receive correlation, even when the correlation is quite large.

Figure 4 shows the performance of the simplified switching scheme compared to the the waterfilling scheme for MISO systems with two or four transmit antennas. The transmit correlation uses the "all equal" model and the correlation is set as $\rho=0.8$. For $M=4$, the SNR threshold was found to be $10.36 \mathrm{~dB}$; for $M=2$, it was $9.44 \mathrm{~dB}$.

\section{CONCLUSIONS}

Orthogonal Space-Time Block Codes (O-STBC) are widely used in MIMO systems to achieve diversity, but the performance of O-STBC over correlated fading channels deteriorates rapidly with increasing channel correlation. With feedback of the channel correlation matrix, the transmitter can employ a linear transformation unit following the STBC encoder to improve the performance. One such scheme chooses the transformation matrix which minimizes the maximum pairwise error probability.

Based on the performance criterion derived in previous work, we use a waterfilling argument to derive a closed 


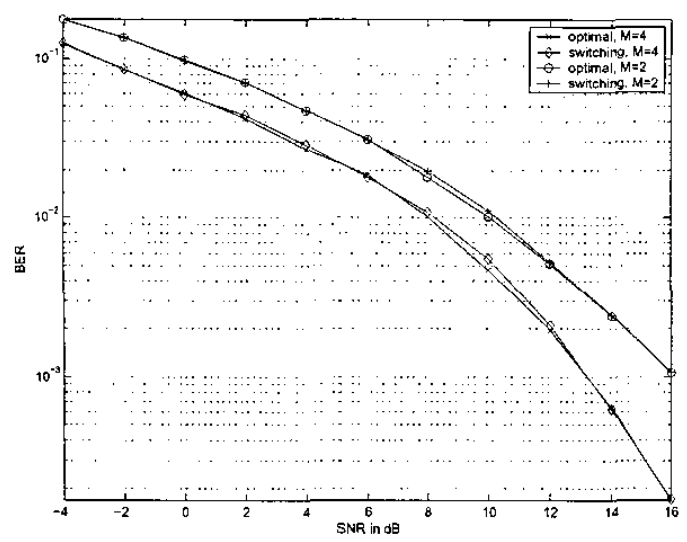

Fig. 4. Switching Scheme vs. Waterfilling.

form solution for the optimal transformation matrix for a MISO system. The same scheme is proven to be optimal for a receive-uncorrelated MIMO system. More generally, for arbitrary MIMO systems, we derive a "second order waterfilling" solution which can be found using numerical algorithms such as Sequential Quadratic Programming.

Finally, two simplified schemes are introduced to reduce the complexity of implementing the optimal technique. One scheme ignores receiver correlation. The other uses STBC or beamforming directly based on the SNR level and channel correlation. They reduce the transmitter complexity dramatically.

\section{APPENDIX}

PROOF OF THEOREM 1

The problem is to choose a non-negative definite matrix $\mathbf{Z}$ to maximize $\operatorname{det}\left[\left(\mathbf{I}_{N} \otimes \mathbf{Z}\right) \eta+\mathbf{R}_{R}^{-1} \otimes \mathbf{R}_{T}^{-1}\right]$ subject to the trace constraint $\operatorname{tr}(\mathbf{Z})=M$. Notice that the correlation matrix $\mathbf{R}_{R}$ and $\mathbf{R}_{T}$ are both positive definite and we can decompose them into the diagonal form,

$$
\begin{gathered}
\mathbf{R}_{T}=\mathbf{U}_{T} \boldsymbol{\Lambda}_{T} \mathbf{U}_{T}^{\dagger}, \text { where } \mathbf{U}_{T} \mathbf{U}_{T}^{\dagger}=\mathbf{I}_{M} \\
\mathbf{R}_{R}=\mathbf{U}_{R} \boldsymbol{\Lambda}_{R} \mathbf{U}_{R}^{\dagger}, \text { where } \mathbf{U}_{R} \mathbf{U}_{R}^{\dagger}=\mathbf{I}_{N} .
\end{gathered}
$$

Then

$$
\begin{aligned}
\operatorname{det} & {\left[(\mathbf{I} \otimes \mathbf{Z}) \eta+\mathbf{R}_{R}^{-1} \otimes \mathbf{R}_{T}^{-1}\right] } \\
& =\operatorname{det}\left[(\mathbf{I} \otimes \mathbf{Z}) \eta+\left(\mathbf{U}_{R} \mathbf{\Lambda}_{R}^{-1} \mathbf{U}_{R}^{\dagger}\right) \otimes\left(\mathbf{U}_{T} \boldsymbol{\Lambda}_{T}^{-1} \mathbf{U}_{T}^{\dagger}\right)\right] \\
& =\operatorname{det}\left[(\mathbf{I} \otimes \mathbf{Z}) \eta+\left(\mathbf{U}_{R} \otimes \mathbf{U}_{T}\right)\left(\boldsymbol{\Lambda}_{R}^{-1} \otimes \mathbf{\Lambda}_{T}^{-1}\right)\left(\mathbf{U}_{R} \otimes \mathbf{U}_{T}\right)^{\dagger}\right] \\
& =\operatorname{det}\left[\left(\mathbf{U}_{R}^{\dagger} \mathbf{I} \mathbf{U}_{R}\right) \otimes\left(\mathbf{U}_{T}^{\dagger} \mathbf{Z} \eta \mathbf{U}_{T}\right)+\mathbf{\Lambda}_{R}^{-1} \otimes \boldsymbol{\Lambda}_{T}^{-1}\right] \\
& =\operatorname{det}\left[\mathbf{I}_{N} \otimes \mathbf{B}+\mathbf{\Lambda}_{R}^{-1} \otimes \mathbf{\Lambda}_{T}^{-1}\right]
\end{aligned}
$$

where $\mathbf{B}=\mathbf{U}_{T}^{\dagger} \mathbf{Z} \eta \mathbf{U}_{T}$. The intermediate steps above come from the fact that [13]

$$
\left(\prod_{i=1}^{N} \mathbf{A}_{i}\right) \otimes\left(\prod_{i=1}^{N} \mathbf{B}_{i}\right)=\prod_{i=1}^{N} \mathbf{A}_{i} \otimes \mathbf{B}_{i} .
$$

The trace constraint is

$$
\begin{aligned}
\operatorname{tr}(\mathbf{B}) & =\operatorname{tr}\left(\mathbf{U}_{T}^{\dagger} \mathbf{Z} \eta \mathbf{U}_{T}\right) \\
& =\operatorname{tr}\left(\mathbf{U}_{T} \mathbf{U}_{T}^{\dagger} \mathbf{Z} \eta\right)=\eta M .
\end{aligned}
$$

Therefore, the problem reduces to finding a non-negative definite matrix $\mathbf{B}$ maximizing $\operatorname{det}\left[\mathbf{I}_{N} \otimes \mathbf{B}+\boldsymbol{\Lambda}_{R}^{-1} \otimes \mathbf{\Lambda}_{T}^{-1}\right]$ with the constraint $\operatorname{tr}(\mathbf{B})=\eta M$.

\section{REFERENCES}

[1] V. Tarokh, H. Jafarkhani, and A. R. Calderbank, "Space-time block code from orthogonal designs," IEEE Trans. Info. Theory, vol. 45, pp. 1456-1467, July 1999.

[2] G. Jongren, M. Skoglund, and B. Ottersten, "Combining beamforming and orthogonal space-time block coding," IEEE Trans. Info. Theory, vol. 48, pp. 611627, March 2002.

[3] H. Sampath and A. Paulraj, "Linear precoding for space-time coded systems with known fading correlations," IEEE Communications Letters, vol. 6, pp. 239 241, June 2002.

[4] S. Zhou and G. B. Giannakis, "Optimal transmitter eigen-beamforming and space-time block coding based on channel correlations," IEEE Trans. Info. Theory, vol. 49, pp. 1673-1690, July 2003.

[5] E. Visotsky and U. Madhow, "Space-time transmit precoding with imperfect feedback," IEEE Trans. Info. Theory, vol. 47, pp. 2632-2639, September 2001.

[6] S. Jafar and A. Goldsmith, "On optimality of beamforming for multiple antenna systems with imperfect feedback," IEEE Proc. ISIT, p. 321, 2001.

[7] R. A. Horn and C. R. Johnson, Matrix Analysis. Cambridge, 1985.

[8] J. P. Kermoal, L. Schumacher, K. I. Pedersen, P. E. Mogensen, and F. Frederiksen, "A stochastic MIMO radio channel model with experimental validation," IEEE Journal on Selected Areas in Communications, vol. 20, pp. 1211-1226, August 2002.

[9] P. E. Gill, W. Murray, and M. H. Wright, Practical Optimization. Academic Press, 1981.

[10] T. M. Cover and J. A. Thomas, Elements of Information Theory. John Wiley, 1991.

[11] D. S. Shiu, G. J. Foschini, M. J. Gans, and J. M. Kahn, "Fading correlation and its effect on the capacity of multielement antenna systems," IEEE Trans. Comm., vol. 48, pp. 502-513, March 2000.

[12] R. Janaswamy, Radiowave Propagation and Smart Antennas for Wireless Communications. Kluwer Academic Publisher, 2001.

[13] A. Graham, Kronecker Products and Matrix Calculus with Applications. Halsted Press,John Wiley and Sons, 1981. 